\title{
Surveying Molecular Vibrations during the Formation of Metal-Molecule Nanocontacts
}

\author{
Lucia Vitali, ${ }^{*}{ }^{\dagger}$ Robin Ohmann, ${ }^{\dagger}$ and Klaus Kern ${ }^{\dagger,}$ \\ ${ }^{\dagger}$ Max-Planck-Institute fuer Festkoerperforschung, Heisenbergstrasse 1, D-70569 Stuttgart, Germany, and ${ }^{\ddagger}$ Institut de \\ Physique de la Matière Condensée, Ecole Polytechnique Fédérale de Lausanne (EPFL), CH-1015 Lausanne, Switzerland
}

\author{
Aran Garcia-Lekue, ${ }^{\S}$ Thomas Frederiksen, ${ }^{\S}$ Daniel Sanchez-Portal, ${ }^{\S, \|}$ and Andres Arnau ${ }^{\S, \|, \perp}$ \\ $\S$ Donostia International Physics Center (DIPC), Paseo Manuel de Lardizabal 4, E-20018 San Sebastian, Spain, \\ "Centro de Fisica de Materiales CFM-MPC, Centro Mixto CSIC-UPV, Apdo. 1072, San Sebastian, Spain, and \\ ${ }^{\perp}$ Departamento de Fisica de Materiales UPV/EHU, Facultad de Quimica, Apdo. 1072, San Sebastian, Spain
}

\begin{abstract}
Molecular junctions have been characterized to determine the influence of the metal contact formation in the electron transport process through a single molecule. With inelastic electron tunneling spectroscopy and first-principles calculations, the vibration modes of a carbon monoxide molecule have been surveyed as a function of the distance from a copper electrode with unprecedented accuracy. We observe a continuous but nonlinear blue shift of the frustrated rotation mode in tunneling with decreasing distance followed by an abrupt softening upon contact formation. This indicates that the presence of the metal electrode sensibly alters the structural and conductive properties of the junction even without the formation of a strong chemical bond.
\end{abstract}

KEYWORDS Inelastic electron tunneling spectroscopy, electron-vibration coupling, single molecule-metal contact, electron conductance, density functional theory, nonequilibrium Green's function, point contact spectroscopy

$\mathrm{C}$ harge transport through metal-molecule systems is a major subject of study in a rapidly growing interdisciplinary research field. It deals with fundamental and applied aspects of science at the nanoscale aiming to control the electron conductance at the molecular level and the uprising of nanotechnology. ${ }^{1,2}$ One of the major results of this research is that the properties of an isolated molecule are not the only fundamental parameters determining the conductance in a metal-molecule junction. Indeed, similarly to the adsorption of molecules on surfaces, the atomistic arrangement at the junction and the coupling between the molecule and the metal electrodes can significantly alter the electronic and structural properties of the molecule. ${ }^{3-5}$ In particular, the stronger the metal-molecule interaction is, the less the measured conductance can be ascribed to molecular properties alone.

Accessing the influence of the molecular contact to the metal in the transport properties is, however, experimentally challenging. Despite the continuous progress, the electron conductance measured using either a scanning tunneling microscope (STM) or a break-junction device ${ }^{6-13}$ has so far not elucidated this question clearly. The strength of the metal-molecule coupling, and in particular its variation during the contact formation, can be characterized through the spectroscopic signal of the molecular vibrations as measured by

* Corresponding author, lucia_vitali@ehu.es

Received for review: 11/10/2009

Published on Web: 01/19/2010 inelastic electron tunneling spectroscopy (IETS). ${ }^{6,9,14-17}$ This can be recorded as a function of the tip-molecule distance allowing understanding to which extent the contact formation influences the molecular properties.

A good molecular prototype to study the influence of the metal bond is carbon monoxide (CO). Indeed, this molecule is used as ligand to contact metal atoms in coordination chemistry and its electronic and vibration properties are very sensitive to the local adsorption environment. Shifts of the vibration frequencies are observed by coadsorption with other gases ${ }^{18}$ and explained by subtle mechanisms of charge transfer. ${ }^{19}$ In the absence of charge transfer, the polarization of the $\mathrm{CO}$ molecule by a proton located at distances much larger than typical bond lengths causes significant shifts in the molecular vibration frequencies. ${ }^{20}$ Therefore, one could expect the $\mathrm{CO}$ molecule on a metal surface to be the ideal candidate to gauge the relation between shifts in the vibration frequency and a progressively varying tip-molecule interaction.

Here, we combine spectroscopic techniques based on scanning tunneling microscopy (STM) with first-principles calculations to achieve a precise characterization of the metal-molecule interaction during the formation of a metal-molecule nanocontact. We use the IETS signal measured at various molecule-metal distances by approaching the tip of an STM to the $\mathrm{CO}$ molecule adsorbed on a $\mathrm{Cu}(111)$ metal surface. Both the measured data and the calculations show characteristic shifts of the vibration modes. In particular, the excitation energy of the frustrated rotation (FR) mode of a single carbon monoxide molecule adsorbed on $\mathrm{Cu}(111)$ changes 
in a nonlinear way in the tunneling regime and softens abruptly upon the formation of the contact with the tip. The behavior of this transverse mode clearly reflects a transition from an attractive to a repulsive tip-molecule interaction passing from the tunneling to the contact regime. This indicates that the structural relaxation at the junction influences the vibration properties as the tip progressively approaches.

The experiments were performed using a home-built scanning tunneling microscope operated at $6 \mathrm{~K}$ in ultrahigh vacuum (UHV) with a base pressure of $1 \times 10^{-11}$ mbar. The $\mathrm{Cu}(111)$ single crystal was cleaned in UHV by cycles of Ar ion sputtering and annealing. Exposing the copper crystal held at $140 \mathrm{~K}$ to 0.5 langmuir of $\mathrm{CO}$ leads to the presence of individual molecules (CO monomers) on the surface. The STM tip, chemically etched from a tungsten wire, was treated in vacuo by electron field emission and soft indentation into the copper surface. This assured a spectroscopic featureless tip near the Fermi energy. Given this preparation, the tip was most likely covered by copper atoms from the substrate.

The conductance of the junction achieved by recording the current at various tip-CO molecule distances under open feedback loop conditions is shown in Figure 1a. As the tip-substrate distance is reduced, the current increases smoothly from the tunneling to the point contact regime following a characteristic exponential dependence with tip-substrate distance. The smooth transition, or more specifically the lack of discontinuities in the current, indicates that the atomic arrangement of the junction was stable at all tip displacements. As point contact is reached, the current exhibits a characteristic plateau with only a weak dependence on distance. The conductance value at point contact is of $\sim 0.1 G_{0}$, where $G_{0}$ is the conductance quantum. Furthermore, topographic images acquired before and after the contact formation confirm that the contact region and the tip have not changed during the formation of the contact. This indicates that the process is fully reversible.

The vibration spectra shown in Figure 1b have been measured along this tip-molecule displacement at the positions indicated by numbers in Figure 1a. In the tunneling regime (blue lines), the vibration spectra are characterized by two features at about 5 and $35 \mathrm{meV}$ that are assigned to the two degenerate transverse vibration modes: the frustrated translation $(\mathrm{FT}(2))$ and the frustrated rotation $(\mathrm{FR}(2))^{16,21}$ (see also Figure 2c). With reduction of the tip-sample distance, an initial shift of about $1.5 \mathrm{meV}$ toward higher energy can be observed in the peak assigned to the FR mode (spectra $1-4$ ). As can be seen in Figure 1c, by a further reduction of the tip distance (spectra 4-7), this trend is reversed. Just before the contact is formed the vibration energy of the FR coincides with the one observed in the initial conditions. As the contact regime is reached (red lines), the FR mode shifts suddenly and sensibly toward lower energies ( $29 \mathrm{meV}$ ), while the FT mode signal loses intensity and broadens. The peak of increasing intensity at about $13 \mathrm{meV}$ (spectra 8 and 9) can be assigned to the Rayleigh mode typical of the $\mathrm{Cu}$ surface. ${ }^{17}$

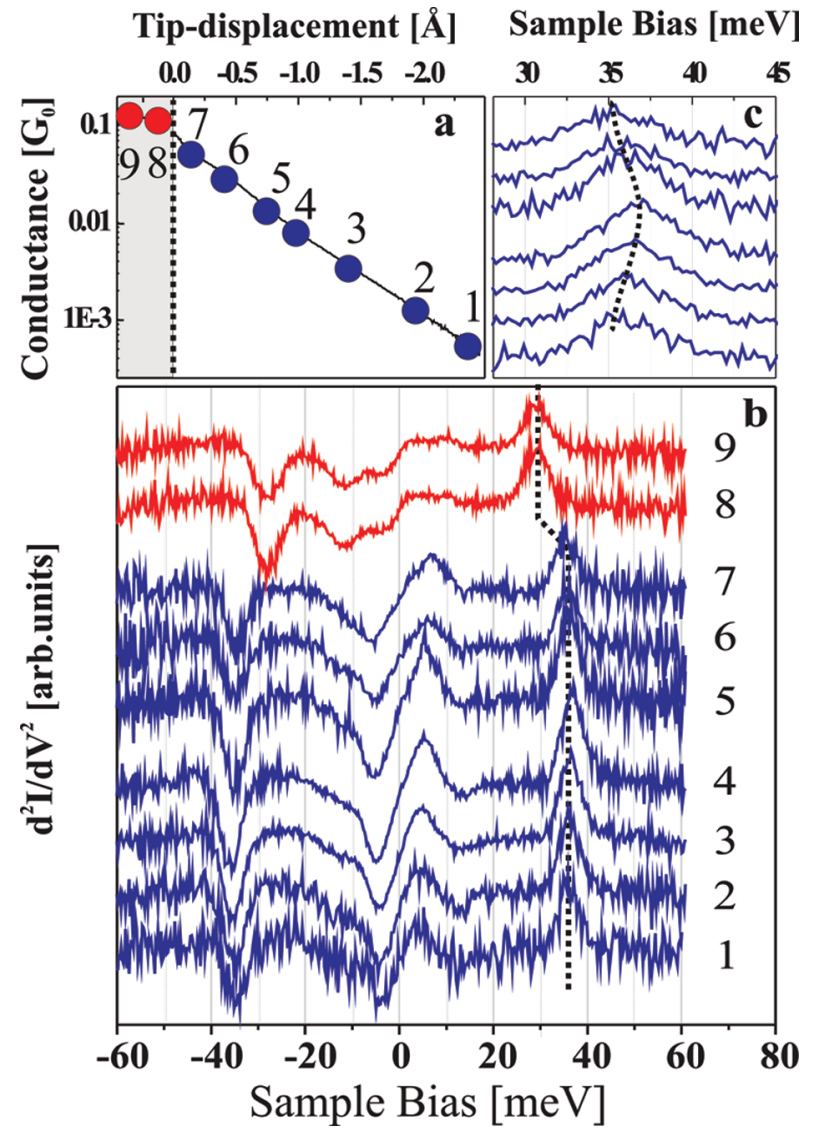

FIGURE 1. Conductance and vibration spectra for individual $\mathrm{CO}$ molecules on a $\mathrm{Cu}(111)$ surface at different tip-substrate distances ranging from tunneling to point contact. (a) Variation of the conductance vs tip displacement. (b) Vibration spectra recorded using a lock-in amplifier (amplitude of the modulation voltage $\Delta V$ $=2.5 \mathrm{meV}$ ) at the positions indicated in (a). The curves are normalized to the tunneling current at the corresponding tip displacement. A vertical offset has been added for a better visualization. (c) Enlarged section of the spectra reported in (b) showing the FR mode achieved in tunneling conditions. The dotted lines in (b) and (c) highlight the shift of the FR mode vs the tip position.

To obtain a physical understanding of the experimentally observed frequency shifts, we have modeled the vibration modes and the inelastic transport using density functional theory (DFT) combined with nonequilibrium Green's function methods. ${ }^{22,23}$ In our calculations the STM tip is modeled by a $\mathrm{Cu}$ adatom adsorbed on a $\mathrm{Cu}(111)$ surface at a face-centered cubic (fcc) hollow site. To simulate the inelastic electron tunneling spectra, we have assumed the adsorption site of $\mathrm{CO}$ on a top-site of $\mathrm{Cu}(111)$, as experimentally observed (scheme in Figure $2 \mathrm{~b}$ ). This overcomes some of the limitations of most local and semilocal DFT functionals which incorrectly give the fcchollow adsorption configuration as the most stable. ${ }^{28,29}$ However, this constrains our predictions for the frequency of the low energy FT modes which becomes imaginary as contact is formed and the system starts to be compressed. This, which would imply an instability of the junction, does not correspond to a real experimental situation. Indeed, the STM images show that the contact area is unmodified. Therefore, in our IETS 


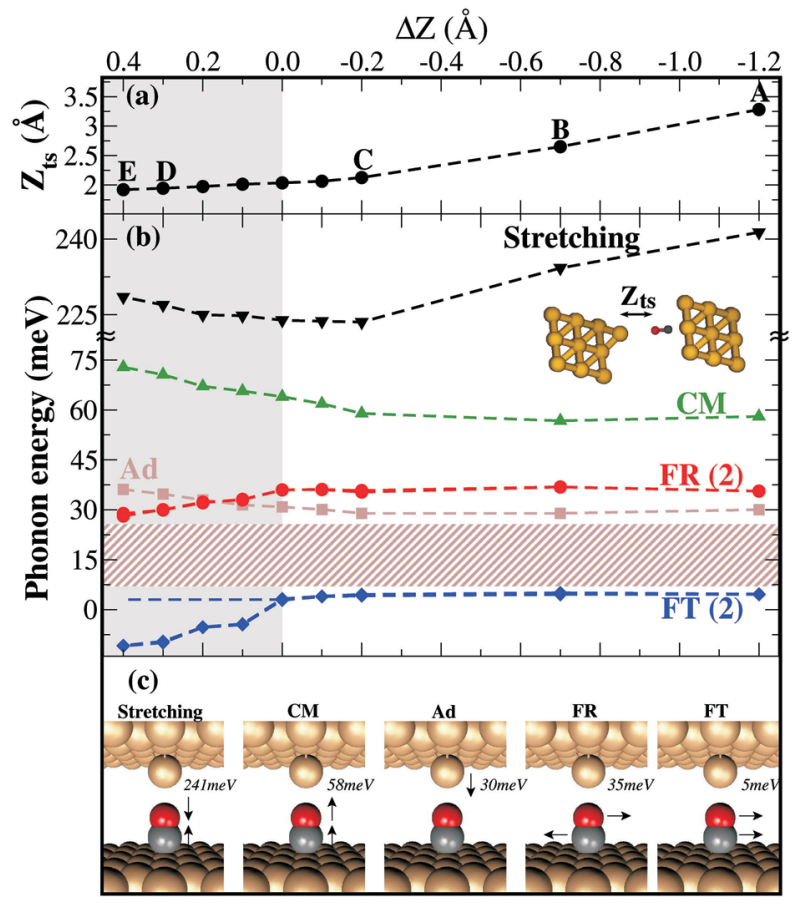

FIGURE 2. (a) Variation of the distance of the tip to the oxygen atom $Z_{\text {ts }}$ with $\Delta Z$ showing structural relaxations at the junction. $\Delta Z$ is defined by the variation in the distance between $\mathrm{Cu}(111)$ planes that are not relaxed during the approach to contact (second layers of the two electrodes). $\Delta Z=0$ corresponds to the instant of contact formation. (b) Evolution of the $\mathrm{CO} / \mathrm{Cu}(111)$ vibration modes. Stretching, $\mathrm{CM}, \mathrm{FR}(2)$, and FT(2) are $\mathrm{CO}$ vibration modes, while $\mathrm{Ad}$ is the tip-apex $\mathrm{Cu}$ adatom longitudinal vibration (see the text). The shaded area covers a region of topmost layer $\mathrm{Cu}$ atoms vibrations. At a tip-sample separation of $Z_{\mathrm{ts}} \sim 2 \AA$ a clear change is seen in the vibration energy of the IETS most active FR mode. The geometry of the junction is also schematically shown. (c) Sketch showing the vibration modes.

simulations, we have kept the FT mode frequency fixed to the last real value ( $3 \mathrm{meV}$ ) prior to contact, in order to analyze the electron-phonon coupling of the FT mode. The FR mode is well-defined for all tip-molecule distances under study.

First, we analyze in detail the vibration modes of the molecular nanocontact. In particular, we focus on the modes localized at the $\mathrm{CO}$ and $\mathrm{Cu}$ adatom, which are shown in panels $\mathrm{b}$ and $\mathrm{c}$ of Figure 2. We observe shifts for all these vibration modes along the transition from tunnel to contact, i.e., a clear indication of the existence of an appreciable tip-sample interaction. $\mathrm{CM}$ and stretching denote the $\mathrm{CO}$ center of mass motion ( $\mathrm{Cu}-\mathrm{CO}$ stretch) and $\mathrm{C}-\mathrm{O}$ stretch, respectively, while $\mathrm{Ad}$ is the $\mathrm{Cu}$ adatom vertical vibration. The shaded area in Figure $2 \mathrm{~b}$ represents the vibration modes of the $\mathrm{Cu}(111)$ surfaces under the $\mathrm{CO}$ molecule and $\mathrm{Cu}$ adatom. It is worth stressing here that not all the vibration modes can be observed experimentally in IETS. Indeed, the CM mode signal has never been reported while the stretching mode is found to induce a lateral motion of the adsorbed $\mathrm{CO}$ molecule, similarly to previously reported results. ${ }^{30}$ Additionally, as the tip-molecule distance is reduced, the frustrated translation mode hybridizes with modes of the tip apex. This explains the experimental observation of a reduced intensity and broadening of these modes as a function of the tip proximity. However, the nature of the FR mode is preserved at all tip-molecule distances making this vibration mode the most suited to survey the formation of the bond.

At large tip-sample distances, in the tunneling regime, the interaction is attractive and reaches its maximum strength well before the instant of contact formation. This, relatively weak, attractive interaction causes (from point A to B) a downward shift of the longitudinal modes (stretching, CM, and $\mathrm{Ad}$ ) and an upward shift of the transverse modes (FR and FT). Correspondingly, a moderate structural relaxation can be observed and the increased distance between the two topmost $\mathrm{Cu}(111)$ layers amounts to $0.05 \AA$. This causes a total energy shift of the FR of about $1.5 \mathrm{meV}$. This trend is reversed at smaller tip-sample distances (from position B to C), in agreement with the experimental observations (Figure 1C). The larger shift of the longitudinal $\mathrm{C}-\mathrm{O}$ stretching mode is partially due to the polarization of the molecular orbitals. ${ }^{20}$

By a further reduction of the electrode separation, we observe an upward shift of the longitudinal modes, which is more pronounced for the CM mode. From this point on, a significant compression of the junction (see Figure $2 \mathrm{a}$ ) and a rather abrupt downward shift of the transverse modes are evident, as soon as point contact is reached. The tip induces a compression of the $\mathrm{Cu}$ atom beneath the CO: the distance of the tip to the oxygen atom $Z_{\text {ts }}$ varies slowly with electrode separation $\Delta Z$ and stays approximately at a value of $\sim 2 \AA$. However, a Mulliken population analysis and projected densities of states onto atomic orbitals of the atoms forming the junction $(\mathrm{Cu}, \mathrm{C}$, and $\mathrm{O})$ at different tip-sample distances reveal that no significant charge transfer or strong covalent $\mathrm{Cu}$-bond formation is present. Therefore, we assign the observed shifts to a modification of the surface potential under the influence of the tip proximity, as the tip-molecule distance is reduced. The interaction between them passes progressively from the attractive to the repulsive regime.

Before making a final comparison between calculated and measured vibration spectra, we consider the conductance spectra shown in Figure 3a. The calculated value of the low bias conductance at the onset of contact formation is $\sim 0.18 G_{0}$. This is slightly larger than the experimentally measured value reflecting the underestimation of the HOMO-LUMO gap in DFT calculations. The existence of this gap implies that the electron conductance occurs in the nonresonant tunneling regime at all tip-sample distances. The two steps observed in the conductance curves at $\sim 5$ and $\sim 35 \mathrm{meV}$ correspond to the excitation of the FT and FR modes, respectively. These steps, which show an increase in the conductance at the onset of the inelastic excitations, indicate the absence of backscattering due to electron-vibration coupling and further substantiate that the conductance occurs in the low-transmission regime. ${ }^{31}$ The corresponding normalized IETS spectra are shown in Figure 3b. The fact that they can be represented in the same $(1 / V)$ scale for all distances indicates small changes in the electronvibration coupling strength. ${ }^{32}$ In the tunneling regime, the 


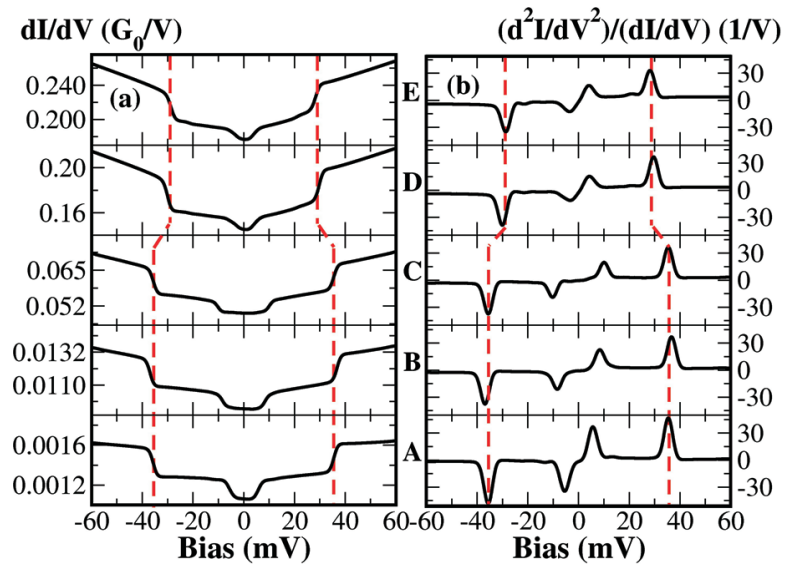

FIGURE 3. Calculated conductance (a) and vibration (b) spectra taken on top of a $\mathrm{CO}$ molecule adsorbed on a $\mathrm{Cu}(111)$ surface at different tip-sample distances shown in Figure 2. A clear shift (red dotted lines) to lower energies is seen in the FR mode where the peak position shifts from 35 to $29 \mathrm{meV}$ as point contact is approached from tunneling.

spectra are dominated by the FT signal at $\sim 5 \mathrm{meV}$ and the FR signal at $\sim 35 \mathrm{meV}$. As the tip approaches contact with the $\mathrm{CO}$ molecule the FT signal spreads out while the FR signal shifts to $\sim 29 \mathrm{meV}$, in agreement with the changes observed in the measured spectra shown in Figure $1 \mathrm{~b}$.

In conclusion, we have shown that by combining highresolution IETS data and first-principles calculations it is possible to monitor the structural and electronic properties of a molecular nanocontact during its formation, i.e., from the tunneling to the contact regime. We find that, even in the case of soft bonding where no significant charge rearrangement across the molecular junction occurs, the changes in the molecular vibrations give evidence of a perturbation of the electronic and structural properties of the junction as the STM tip approaches a single molecule. Indeed, changes of the low energy vibration modes of the metal-molecule are excellent fingerprint of the presence of other metallic electrodes like the apex of the STM tip. The observation of their shift is therefore a powerful tool to monitor the formation of a metallic nanocontact. As the tip of the STM can be more widely understood as the presence of any metallic electrode, we believe that these results have a general validity to the measurements of conductance through molecular junctions. The results reported here might therefore clarify a few of the uncertainties in the electron transport through nanometer scale junctions.

Acknowledgment. We thank Magnus Paulsson and Talat Rahman for stimulating discussions. Support from the Basque Departamento de Educacion, UPV/EHU (Grant No. IT-366-07), the Spanish Ministerio de Ciencia e Innovacion (Grants No. FIS2007-6671-C02-00, and MAT2007-62732), the ETORTEK program funded by the Basque Departamento de Industria and the Diputacion Foral de Guipuzcoa, and the Danish FNU (Grant No. 272-07-0114) are gratefully acknowledged.

\section{REFERENCES AND NOTES}

(1) Tao, N. J. Nat. Nanotechnol. 2006, 1, 173.

(2) Moth-Poulsen, K.; Bjornholm, T. Nat. Nanotechnol. 2009, 4, 551.

(3) Jang, S.-Y.; Reddy, P.; Majundar, A.; Sagelman, R. A. Nano Lett. 2006, 6, 2362 .

(4) Chang, Y.; Zwolak, C. M.; di Ventra, M. Nano Lett. 2004, 4, 1709

(5) Seitz, O.; Vilan, A.; Cohen, C.; Chan, C.; Hwang, J.; Kahn, A.; Cahen, D. J. Am. Chem. Soc. 2007, 129, 7494.

(6) Stipe, B. C.; Rezai, M. A.; Ho, W. Science 1998, 280, 1732

(7) Agraït, N.; Untiedt, C.; Rubio-Bollinger, G.; Vieira, S. Phys. Rev. Lett. 2002, 88, 216803.

(8) Venkataraman, L.; Lare, J. E.; Nuckolls, C.; Hybertsen, M. S.; Steigerwald, M. L. Nature 2006, 442, 904

(9) Tal, O.; Kiguchi, M.; Thijssen, W. H. A.; Djukic, D.; Untiedt, C.; Smit, R. H. M.; van Ruitenbeek, J. M. Phys. Rev. B 2009, 80, No. 085427.

(10) Joachim, C.; Gimzewski, J. K.; Schlittler, R. R.; Chavy, C. Phys. Rev. Lett. 1995, 74, 2102

(11) Vitali, L.; Ohmann, R.; Stepanow, S.; Gambardella, P.; Tao, K.; Huang, R.; Stepanyuk, S. V.; Bruno, P.; Kern, K. Phys. Rev. Lett. 2008, 101, 216802 .

(12) Schulze, G.; Franke, K. J.; Gagliardi, A.; Romano, G.; Lin, C. S.; Rosa, A. L.; Niehaus, T. A.; Frauenheim, Th.; Di Carlo, A.; Pecchia, A.; Pascual, J. I. Phys. Rev. Lett. 2008, 100, 136801.

(13) Limot, L.; Kroeger, J.; Berndt, R.; Garcia-Lekue, A.; Hofer, W. A. Phys. Rev. Lett. 2005, 94, 126102.

(14) Vitali, L.; Burghard, M.; Wahl, P.; Schneider, M. A.; Kern, K. Phys. Rev. Lett. 2006, 96, No. 086804.

(15) Pascual, J. I.; Lorente, N.; Song, Z.; Conrad, H.; Rust, H. P. Nature 2003, 423, 525 .

(16) Lauhon, L. J.; Ho, W. Phys. Rev. B 1999, 60, 8525(R).

(17) Vitali L., Borisova S. D., Rusina G. G., Chulkov E. V., Kern K., submitted.

(18) Yamamoto, I.; Nanba, T. Surf. Sci. 1988, 202, 377.

(19) Blyholder, G. J. Phys. Chem. 1964, 68, 2772.

(20) Lupinetti, A. J.; Fau, S.; Frenking, G.; Strauss, S. H. J. Phys. Chem. A 1997, 101, 9551

(21) Lorente, N.; Ueba, H. Eur. Phys. J. D 2005, 35, 341

(22) Paulsson, M.; Frederiksen, T.; Ueba, H.; Lorente, N.; Brandbyge, M. Phys. Rev. Lett. 2008, 100, 226604.

(23) The SIESTA and TranSIESTA ${ }^{24,25}$ packages were used for the DFT calculations. Periodic boundary conditions on unit cells consisting of one $\mathrm{CO}$ molecule and one $\mathrm{Cu}$ tip atom together with $54 \mathrm{Cu}$ atoms (six layers of $3 \times 3$ ) for the $\mathrm{Cu}(111)$ surfaces were employed. Atomic coordinates of the molecule, tip, and surface copper atoms (vibration region) were relaxed until forces $<0.02 \mathrm{eV} / \mathrm{A}$. A single-zeta plus polarization and a split-valence double-zeta plus polarization basis sets were used for the $\mathrm{Cu}$ atoms and $\mathrm{CO}$, respectively, as well as the generalized gradient approximation for exchange-correlation, a cutoff of 200 Ry for the real-space grid integrations, and the $\Gamma$-point approximation for sampling the three-dimensional Brillouin zone. Vibration frequencies were obtained diagonalizing the dynamical matrix extracted from finite differences, for atoms in the vibration region. ${ }^{22}$ The inelastic contribution to the conductance was calculated employing the lowest order expansion approximation described in refs 26 and 27.

(24) Soler, J. M.; Artacho, E.; Gale, J. D.; Garcia, A.; Junquera, J.; Ordejon, P.; Sanchez-Portal, D. J. Phys.: Condens. Matter 2002, 14,2745 .

(25) Brandbyge, M.; Mozos, J. L.; Ordejon, P.; Taylor, J.; Stokbro, K. Phys. Rev. B 2002, 65, 165401

(26) Paulsson, M.; Frederiksen, T.; Brandbyge, M. Phys. Rev. B 2005 , 72,201101

(27) Frederiksen, T.; Paulsson, M.; Brandbyge, M.; Jauho, A.-P. Phys. Rev. B 2007, 75, 129901

(28) Lopez, N.; Nørskov, J. K. Surf. Sci. 2001, 477, 59

(29) Alcántara-Ortigoza, M.; Heid, R.; Bohnen, K.-P.; Rahman, T. S. Phys. Rev. B 2009, 79, 125432

(30) Komeda, T.; Kim, Y.; Kawai, M.; Persson, B. N. J.; Ueba, H. Science 2002, 295, 2055.

(31) Frederiksen, T.; Lorente, N.; Paulsson, M.; Brandbyge, M. Phys. Rev. B 2007, 75, 235441.

(32) Teobaldi, G.; Peñalba, M.; Arnau, A.; Lorente, N.; Hofer, W. A. Phys. Rev. B 2007, 76, 235407. 